\title{
Comparing outcomes of patients submitted to coronary artery bypass graft surgery accompanied in a general or cardiac Intensive Care Unit
}

Beatriz Eva Pires ${ }^{1}$, Clesnan Mendes-Rodrigues*1, Fabiola Alves Gomes ${ }^{1}$, Rosângela de Oliveira Felice ${ }^{2}$, Iolanda Alves Braga $^{2}$, Omar Pereira de Almeida Neto ${ }^{1}$, Lívia Maria Ambrósio da Silva ${ }^{2}$

${ }^{1}$ Nursing, Medicine Faculty, Federal University of Uberlândia, Uberlândia, Brazil

${ }^{2}$ Clinical Hospital of Uberlândia, Federal University of Uberlândia, Uberlândia, Brazil

Received: July 17, 2018

DOI: $10.5430 /$ cns.v7n1p39
Accepted: September 4, 2018

Online Published: September 11, 2018

\begin{abstract}
Background: Cardiovascular diseases are the main cause of death and loss of quality of life in the long term. In the USA, cardiac surgeries represent the most performed surgery category, with an average cost of USD 40,000. Because it is a complex procedure and leads the patient to a critical post-operative state, there is a necessity of intensive care.

Objective: Compare the health indicators of patients submitted to postoperative myocardial revascularization surgery when they are in a general Intensive Care Unit (GICU) or in a cardiac Intensive Care Unit (CICU) in a Brazilian institution.

Methods: This is a quantitative, retrospective, descriptive study of patients submitted to a coronary artery bypass grafting surgery in a hospital of high complexity. Data were collected from the medical records and grouped into two phases. Phase 1 is the pre-implantation of the CICU, with a total of 50 patients, in a period in which the postoperative of myocardial revascularization surgeries were performed in the GICU. The second phase corresponds to the postoperative period performed in the CICU, with a total of 60 patients.

Results: Males were predominant in the study, with the mean age being over 60 years. The most frequent comorbidities in the two groups were systemic arterial hypertension, dyslipidemias and type 2 diabetes mellitus. The main postoperative complications were surgical site infections, cardiac arrhythmias and pleural effusion. After the implementation of the specialized ICU, there was a reduction in the total hospitalization time and a reduction in cost by day and total cost of hospitalization.

Conclusions: The specialized ICU promoted a reduction in the total hospitalization time of patients submitted to coronary artery bypass grafting surgeries and a reduction in hospital costs for the Brazilian public health service, facts that demonstrate the importance of these specialized services in high complexity hospitals.
\end{abstract}

Key Words: Hospital costs, Cardiac surgery, Intensive Care Unit

\section{INTRODUCTION}

Cardiovascular diseases are the main cause of death and loss of quality of life in the long term, representing more than $30 \%$ of all deaths with known cause. In the world, they are responsible for about 12 million deaths a year, considering that about $60 \%$ of deaths due to cardiovascular diseases occur in developing countries. ${ }^{[1]}$

Considering the cardiovascular disease as a disease of great

\footnotetext{
*Correspondence: Clesnan Mendes-Rodrigues; Email: clesnan@hotmail.com; Address: Enfermagem, Faculdade de Medicina, Universidade Federal de Uberlândia, Uberlândia, Av. Pará 1720, CEP 38402-902, Brazil.
} 
impact, it is possible to observe an epidemiological transition from demographic, economic and health aspects in developing countries like Brazil, ${ }^{[2]}$ thus anticipating a global epidemic of this disease. In addition, this cardiovascular disease is one of the main causes of prolonged hospital stay, which consequently leads to a hospitalization with higher expenses for health services. Among the methods for treatment of coronary artery diseases, coronary artery bypass graft surgery (here treated as myocardial revascularization) is suggested and is characterized by high costs, being increasingly performed in recent years. In the United States of America, cardiac surgeries represent the most performed surgery category, with a mean hospitalization cost of USD 40,000. Annually, the cost of cardiac surgical procedures is more than USD 20 billion, which represents from $1 \%$ to $2 \%$ of the country's health expenses. ${ }^{[3,4]}$ Because it is an extremely invasive procedure, which exposes the patient to high risks, the myocardial revascularization surgery is only performed when there is no possibility to treat the injuries through other coronary interventions. ${ }^{[5]}$

Considering the myocardial revascularization surgery is a highly complex procedure, its results have important organic repercussions altering several physiological mechanisms of the patients. These repercussions could lead to a critical state in the postoperative period, which in turn implies the need of intensive care unit (ICU) and healthcare. Also are required qualified and prepared professionals for the management of these patients, in order to establish a good recovery and discharged. Immediate postoperative care is extremely important to guarantee the patient a good recovery and rehabilitation, since the complications that represent greater risks to the patient occur during the first hours of the postoperative period. ${ }^{[3,6]}$

In this way, in order to reduce the risks of complications and to offer intensive and full-time care, the postoperative period of myocardial revascularization surgery should take place in an ICU environment, whether it is specialized in cardiac patients or a general unit. Specialized neurological ICU's have better performance when compared with a general ICU (GICU)'s, ${ }^{[7]}$ but for common diseases the specialized ICU's did not show clear differences. ${ }^{[8]}$ Thus, it is necessary to question and evaluate whether the postoperative care of myocardial revascularization surgery performed in a cardiac specialized ICU (CICU) would have better results than when performed in a GICU. Factors to be considered are related to the length of hospital stay, postoperative complications and financial costs of the procedures for the public health system.

The objective of this study was to compare the health outcomes of patients submitted to myocardial revascularization surgery in the postoperative period when the postoperative was performed in a GICU or in a CICU.

\section{MATERIALS AND METHODS}

\subsection{Study design}

This is a quantitative and retrospective study performed in a general university hospital of high complexity, with 535 beds, including 30 GICU beds and 7 CICU beds. The data were collected from the patient records, and then grouped in two phases. The first phase refers to the pre-implantation of the CICU, a period in which the postoperative care myocardial revascularization surgeries were performed in the GICU from August 2012 to August 2014. The second phase refers to the period when the postoperative cares for surgeries were performed in the CICU, and goes from October 2014 to April 2016.

The two phases are comparable since in both phases the surgical procedures were performed by the same surgical team and in the same surgical center. The main difference in the two phases was the post-surgery care, which was held in a GICU prior to October 2014. From this date, all patients were accompanied in CICU with a team of physicians specialized in cardiology and nursing previously and continuously trained to care for cardiologic patients. We were not able to measure the profile of these professionals during these phases because it was a retrospective study.

\subsection{Ethical considerations}

The study was approved by the Ethics and Research Committee of the Federal University of Uberlândia ("Universidade Federal de Uberlândia"), State of Minas Gerais, Brazil, reference number 942170; according to the Declaration of Helsinki. There was no external source of financing or conflict of interests.

\subsection{Inclusion and exclusion criteria}

In the study, we included patients of both genders, over the age of 18 years, who were submitted exclusively for myocardial revascularization surgery. Patients with incomplete medical records or the ones that had other cardiac surgical interventions in the same period were excluded.

\subsection{Data collection}

In the GICU, 50 patient records were analyzed, and in the CICU, 60 patient records were analyzed, for a total of 110 patient records. To characterize patients' profiles, we recorded sex, age, cardiopulmonary bypass time, days of hospitalization, pre-ICU stay time, ICU stay time, post-ICU stay time; main postoperative complications; EURO Score, and the costs of each patient for the public health system during 
hospitalization time. In the public health system in Brazil the hospitals are paid relative to hospital stay time spent in each type of unit and in some cases, there is additional payment due to more specialized procedures. All costs were transformed to USD, based on the quotation of the day of discharge. The costs here reflect the costs paid to the hospital by the public health system from Brazil and do not reflect the real costs of hospitalization.

\subsection{Statistical analysis}

The dichotomous data were evaluated by relative and absolute frequencies and compared between the two phases by independent Chi-Square tests (for variables with all expected frequencies greater than 5) or Fisher's Exact test (for variables with at least one expected frequency less than 5). The quantitative data were evaluated for the presence of normality by the Kolmogorov-Smirnov Lilliefors test, and all of analyses showed absence of Gaussian distribution. Therefore, the data were presented as median, minimum and maximum values and interquartile range; and the Median test and Mann Whitney test were used to compare the two phases. Quantitative data were also correlated with Spearman's correlation and significance was tested with Student's $t$-test.

Additionally, the total cost and cost per day of patients were also compared with ANCOVA using the preoperative stay time, ICU stay time and the post-operative stay time as covariates. This analysis was based on the payment of costs as a function of time in each period of hospitalization (inpatient or ICU), and it was not possible to separate the cost for each one of the hospitalization periods.

For all analyses a significance level of .05 was adopted.

\section{RESULTS}

A total of 110 patients were included in the study. In the GICU, 50 patients were evaluated, with a median age of 62 years, of which 37 (74\%) were male. In the CICU, 60 patients were evaluated, with a median age of 61 , of which $47(78 \%)$ were male.

Among the comorbidities, the most evident in the two groups were systemic arterial hypertension, dyslipidemias and type 2 diabetes mellitus and smoking. No significant differences between the phases were found, except that chronic obstructive pulmonary disease was more prevalent in patients from the CICU with $16.67 \%$ versus $2.00 \%$ in the GICU ( $p=.025)$ (see Table 1). The Euro SCORE was the same in the two phases, with a median value for the first group of $1.87 \%$ of risk of death in the GICU and $1.69 \%$ for the CICU ( $p=.566)$. The age and the cardiopulmonary bypass time also were the same in the two phases (see Table 1).

In this way, we could verify that the complications in the post-operative period were the same in the GICU and CICU (see Table 2). The ones with greater incidence of complications included surgical site infections, cardiac arrhythmias and pleural effusion, with no significant difference between the groups or phases (see Table 2).

Table 1. Baseline and comorbidities comparison of patients undergoing myocardial revascularization surgery and treated in a GICU or CICU

\begin{tabular}{llll}
\hline Trait & General ICU, \% (n) & Cardiac ICU, \% (n) & $p$ \\
\hline Sex: Male & $74.00(37)$ & $78.33(47)$ & $.759^{\Phi}$ \\
$\quad$ Female & $26.00(13)$ & $21.67(13)$ & $1.000^{\Phi}$ \\
Systemic Arterial Hypertension & $86.00(43)$ & $85.00(51)$ & $.7400^{\Phi}$ \\
Dyslipidemia & $50.00(25)$ & $55.00(33)$ & $1.000^{\Phi}$ \\
Diabetes Mellitus Type 2 & $44.00(22)$ & $43.33(26)$ & $1.000^{\Phi}$ \\
Congestive Heart Failure & $4.00(2)$ & $3.33(2)$ & $.455^{\&}$ \\
Chagas Disease & $2.00(1)$ & $0.00(0)$ & $.025^{\Phi}$ \\
Chronic Obstructive Pulmonary Disease & $2.00(1)$ & $16.67(10)$ & $.392^{\Phi}$ \\
Smoking & $40.00(20)$ & $50.00(30)$ & $.465^{\&}$ \\
Chronic Renal Insufficiency & $10.00(5)$ & $5.00(3)$ & $\boldsymbol{p}$ \\
\hline Trait (unit) & Median (Minimum - Maximum), IQR & $.848^{\ddagger}$ \\
\hline Age (years) & $62(45-76), 14$ & $61(42-81), 10.25$ & $.958^{\ddagger}$ \\
Cardiopulmonary bypass time (min) & $68(0-120), 20$ & $70(0-115), 26.25$ & $.566^{\ddagger}$ \\
EURO Score (\%) & $1.87(0.88-5.78), 1.03$ & $1.69(0.58-5.57), 1.38$ & \\
\hline
\end{tabular}

Note. ${ }^{\Phi}$ : probability based on the Qui-square test of independence; ${ }^{\&}$ : probability based on Fisher's exact test; ${ }^{\ddagger}$ : probability based on Median test; IQR: interquartile range 
Table 2. Comparison of postoperative complications of myocardial revascularization surgery for patients treated in a GICU or CICU ( $\mathrm{n}=50$ in GICU and $\mathrm{n}=60$ in CICU)

\begin{tabular}{llll}
\hline Complications & General ICU, \% (n) & Cardiac ICU, \% (n) & $p$ \\
\hline Surgical Site Infection & $30.00(15)$ & $18.33(11)$ & $.227^{\Phi}$ \\
Cardiac Arrhythmia & $8.00(4)$ & $15.00(9)$ & $.403^{\Phi}$ \\
Pleural Effusion & $6.00(3)$ & $6.67(4)$ & $1.000^{\Phi}$ \\
Acute renal failure & $2.00(1)$ & $5.00(3)$ & $.624^{\&}$ \\
Pneumonia & $10.00(5)$ & $1.67(1)$ & $.090^{\&}$ \\
Atelectasy & $2.00(1)$ & $6.67(4)$ & $.374^{\&}$ \\
Coagulation Disorder & $2.00(1)$ & $0.00(0)$ & $.455^{\&}$ \\
Death in the ICU & $2.00(1)$ & $5.00(3)$ & $.654^{\&}$
\end{tabular}

Note. ${ }^{\Phi}$ : probability based on Qui-square test of independence; ${ }^{\circledR}$ : probability based on Fisher's exact test

Table 3. Length of hospital stay in each operative phase and patient costs for the public health system for patients with myocardial revascularization surgery treated in a GICU or CICU

\begin{tabular}{llll}
\hline \multirow{2}{*}{ Trait (unit) } & \multicolumn{2}{c}{ Median (Minimum - Maximum), IQR } & $\boldsymbol{p}$ \\
\cline { 2 - 4 } & GICU $(\mathbf{n}=\mathbf{5 0})$ & CICU (n= 60) & .454 \\
\hline Pre ICU stay (day) & $20.5(2-79), 21.75$ & $4(1-22), 2$ & .101 \\
ICU stay (day) & $4(2-11), 2$ & $4(0-48), 4.25$ & .071 \\
Post ICU stay (day) & $6(0-81), 6.75$ & $27.5(11-87), 20.25$ & .130 \\
Hospital stay (day) & $35(6-143), 27$ & $5,311.43(5,726-1,0451), 2,188$ & $<.001$ \\
Cost (USD) & $8,758.84(8,625-18,150), 2,033$ & .338 \\
Cost by day (USD) & $240.56(88-1,446), 139$ & $214.59(66-655), 158$ & \\
\hline
\end{tabular}

Note. IQR: interquartile range; $p$ : probability based in Median test

When comparing the median, the hospitalization times in each ICU phase (pre-ICU, ICU and post-ICU) and the values regarding the cost by day during the patient's hospitalization for the public health system, there are no differences in the median of the two phases (see Table 3). Only the cost of hospitalization shows differences in the phases, with the GICU showing a higher cost to the public health system, USD 8,758.84 versus USD 5,311.43 in the CICU $(p<.001$, see Table 3).

However, when comparing the mean rank sum (a distribution indicator), the length of ICU stay was 48.19 in the GICU and 61.59 in the CICU $(p=.025)$. For the post-ICU stay time the GICU showed a mean rank sum of 63.9, while in the CICU it was $48.50(p=.011)$. The hospital stay also was significantly different between the two phases, since, for the GICU the mean rank sum was 63.50, and for the CICU it was 48.83 ( $p$ $=.016$ ). As the mean rank shows the groups with the higher values, these results show that while the CICU increases the number of patients with more stay time in the ICU, it decreases the stay time in the post-ICU and in total hospital stay. Probably the stay in CICU permits more stabilization and decreases complications in the post-ICU.
As the costs are paid in relation to the time in each unit, the total and daily cost was corrected considering the preICU, ICU and post-ICU stay times. This cost was different for each ICU $\left(F_{1,105}=52.06 ; p<.001\right)$, and the covariates were significant for the pre-ICU stay time $\left(F_{1,105}=5.32\right.$, $p=.023)$, for the ICU stay time $\left(F_{1,105}=6.18, p=\right.$ $.014)$, and for the post-ICU stay time $\left(F_{1,105}=16.04\right.$; $p<.001)$. The mean corrected cost was USD 8,624.78 (CI95\%: 8,057.06; 9,192.51) for the GICU and USD 5,726.37 (CI95\%: 5,211.94; 6,240.80) for the CICU. The corrected daily cost was also different for the two ICU's $\left(F_{1,105}\right.$ $=18.05 ; p<.001)$, with the pre-ICU stay time $\left(F_{1,105}=\right.$ $52.21 ; p<.001)$ and the post-ICU stay time $\left(F_{1,105}=21\right.$; $p<.001)$, and not significant for the ICU stay time $\left(F_{1,105}\right.$ $=0.01 ; p=.922)$. The mean corrected daily cost was USD 316.00 (CI95\%: $277.30 ; 354.71$ ) for the GICU and USD 199.64 (CI95\%: 164.57; 234.71) for the CICU.

The Euro Score was positively correlated with patient age $\left(r_{s}=0.578, p \leq .001\right)$, ICU stay time $\left(r_{s}=0.310, p=.001\right)$, Cardiopulmonary bypass time $\left(r_{s}=0.272, p=.004\right)$, hospital stay time $\left(r_{s}=0.244, p=.010\right)$, and cost $\left(r_{s}=0.213\right.$, $p=.025)$. The other correlations were not significant or show 
mathematical association (stay time and cost).

Regarding the mortality rate, of the 50 patients evaluated in the GICU there was 1 death (2\%), and of the 60 patients in the CICU, there were 3 deaths $(5 \%)$, with no significant difference among the phases $(p=.624)$.

\section{Discussion}

Most patients in the study are male, with a mean age of 60 years. This profile is in agreement with the literature, which shows that the epidemiology of coronary diseases is predominant in a population over 60 years of age and male. ${ }^{[9-11]}$ The main comorbidities evidenced were systemic arterial hypertension, dyslipidemia and type 2 diabetes mellitus. In a population with coronary artery disease, which usually presents these underlying diseases, this patient profile was already expected, since studies show that dyslipidemias, systemic arterial hypertension, type 2 diabetes mellitus, smoking and obesity are the major risk factors for coronary artery diseases. ${ }^{[12-15]}$

Type 2 diabetes mellitus affects approximately $25 \%$ of patients diagnosed with acute myocardial infarct. ${ }^{[16,17]}$ The increase in the number of systemic arterial hypertension, diabetes mellitus type 2 and dyslipidemias is mainly related to the lifestyle of the population. Studies show that in the United States, due to changes in lifestyle, there will be a $10 \%$ increase in cardiovascular diseases between 2010 and 2030. ${ }^{[1]}$

Still regarding the clinical profile, the EURO score median of patients that were in the GICU (1.87) was higher than the CICU (1.69), but not significantly different. There is an important role for accurate prediction models of risk (such as EURO score) in the current practice of cardiac surgery. These models allow surgeons and institutions to compare results in a significant way. They are also useful in surgery decision making, development of preoperative informed consent, quality and management of health care. It was designed to provide a post-surgery mortality prediction of up to 30 days in patients undergoing cardiac surgery. It has also been used to provide other useful parameters, including long-term mortality, length of stay in ICU, complications and costs in cardiac surgery, ${ }^{[18]}$ as some findings here. The use of this score showed that the quality and management of patient care in a specific ICU provide better clinical outcomes and reduce complications and hospital costs.

Regarding complications, there were perceived high rates of surgical site infection and pneumonia in patients hospitalized in the GICU when compared of CICU. The specific ICU can allow the nurse to perform health care practice more efficiently, identifying and addressing the specific needs of

Published by Sciedu Press each patient and in the best possible way. ${ }^{[19]}$ The CICU can be a positive predictor for the nurse to perform scientific methods of cardiovascular care that reduce surgical site infection, providing subsidies for the development of healthcare plans, implementation of interventions and evaluation in accordance with real needs. ${ }^{[2]}$ The GICU has a mixture of patients that would lend a higher risk to post-operative patients overall, once the patients are exposed to different agents. The spatial separation of the patients can corroborate for a reduction of local fauna of micro-organisms and a better strategy for prevention of health care related infections based on this profile. Unfortunately we do not evaluate the profile of infections and micro-organisms of the two units, besides the impact of the presence of other patients in the GICU. Although our findings have not detected differences in the number of infections in both units for these patients.

Considering the cardiovascular surgeries are the most effective therapy, there is an increase in the number of these procedures, which are of high cost for the health services. Thus, considering the high costs of these procedures, which are related to the high hospitalization time, there is always the quest for methods that may contribute to its reduction. In the present study, we verified the reduction of the cost of hospitalization and cost by day after the implementation of the CICU, a fact that was also observed in another study with specialized ICUs in 2009. ${ }^{[8]}$

The lowest total cost may be related to the reduction in the total time of hospitalization of the patients, since the shorter hospitalization time will reduce the patient's expenses for the service. We did not find differences in this average but found that the CICU patients showed lower times than patients from GICU, when time distributions were compared. The positive impacts of the CICU are due to the fact that in these units there are specific professionals and materials to treat a defined pathology, which can lead to better management of the cases. ${ }^{[8]}$ Studies show that the optimization of care provided by a trained intensivist team positively influences the reduction of hospitalization time. ${ }^{[3]}$ However, the literature shows in a prior study that there are no consistent benefits that can exalt the advantages of a specialized ICU in relation to a GICU, and that there is a necessity of further studies on this topic, since the implementation of a specialized unit is of high financial impact. ${ }^{[20]}$

After the implantation of the CICU, there was a reduction in preoperative hospitalization time, post-operative hospital stay and total time of hospital stay. However, the length of stay in the ICU remained the same in both periods, with some differences in time distribution. The reduction in preoperative hospitalization may be related to the fact that the 
cardiologic unit has a greater number of specific postoperative beds for cardiac surgery, providing a greater number of surgeries, which could not happen in the GICU.

The same median stay period in the GICU and CICU may have been similar due to the fact that there is the same demand, with similar comorbidities and diagnoses, so the length of hospitalization may be related not exclusively to postoperative complications. In addition to the postoperative complications, the preoperative condition of the patient may be a factor that may prolong the length of stay in intensive care and, consequently, a longer hospitalization. ${ }^{[11]}$

With the specialized unit equipped with human resources and specific materials for the postoperative period of myocardial revascularization, the patient tends to be discharged more hemodynamically compensated and with a lower risk of complications, which would also reduce the length of hospital stay after surgery and after the ICU period. A patient with fewer complications generates less hospital costs, considering that the costs of the cardiac surgeries are directly related to the complications, which may impact the hospitalization time. ${ }^{[4]}$

A study comparing a trauma specialized unit and a nonspecialized unit showed that the specialized units presented better results in several aspects, mainly in the reduction of postoperative complications and also allowed the accomplishment of a greater number of surgeries and without significant difference in mortality rate. ${ }^{[21]}$ However, another study with patients diagnosed with intra-cerebral hemorrhage showed a reduction in the mortality rate when patients were admitted to a specialized unit. This reduction was related to the fact that in these units they have full-time intensive care physicians which improves the handling of cases, resulting in better outcomes. ${ }^{[22]}$

As described previously, the literature shows that a specialized unit allows a better handling of the cases because it has a team with professionals trained for a specific area and with specific material resources, which could possibly lead to a reduction in length of hospital stay and a reduction of financial costs for health service as evidenced in this present study. This also shows that additional studies are necessary, mainly comparing the healthcare offered in these two types of ICU and comparing different hospitals for more ample generalizations.

\section{Conclusions}

The present study allowed the conclusion that there was no significant statistical difference in the number of postoperative complications of myocardial revascularization surgeries among patients admitted to the GICU and to the cardiology specialized ICU.

However, patients who were admitted to the CICU postoperative of myocardial revascularization surgery had a decrease in hospital costs for the health service when compared to those ones admitted to GICU. This reduction in hospitalization cost and time allows optimizing the management of resources available to the health service and a greater turnover of patients, with more surgeries. These factors demonstrate the importance of the specialized service in hospitals, but a greater number of studies are needed on the topic, considering that the literature has few studies that show the relevance of specialized ICU's in health services.

\section{ACKNOWLEDGEMENTS}

The original data used in this study come from the specialization work defended by Beatriz Eva Pires as Resident Nurse in Attention to Critical Care Patients of the Program of Residency in the Professional Area of Health (Uniprofessional and Multiprofessional) of the Medicine Faculty of the Federal University of Uberlândia.

\section{Conflicts of Interest Disclosure}

The authors declare they have no conflicts of interest.

\section{REFERENCES}

[1] Havranek EP, Mujahid MS, Barr DA, et al. Social determinants of risk and outcomes for cardiovascular disease: a scientific statement from the American Heart Association. Circulation. 2015; 132: 873898. PMid: 26240271. https://doi .org/10.1161/CIR. 000000 0000000228

[2] Ribeiro KRA. Pós-operatório de revascularização do miocárdio: complicações e implicações para enfermagem [Post-operative myocardial revascularization: complications and implications for nursing]. Revista de Pesquisa: Cuidado é Fundamental Online. 2018; 10(1): 254-259.

[3] Stephens RS, Whitman GJ. Postoperative critical care of the adult cardiac surgical patient. Part I: routine postoperative care. Critical Care Medicine. 2015; 43: 1477-97. PMid: 25962078. https: //doi.org/10.1097/CCM.0000000000001059

[4] Cheng DCH. Fast track cardiac surgery pathways: Early extubation, process of care, and cost containment. Journal of Cardiothoracic and Vascular Anesthesia. 1998; 88: 1429-33. PMId: 9637632. Available from: http://anesthesiology.pubs.asahq.org/ar ticle.aspx?articleid=1947431

[5] Hillis LD, Smith PK, Anderson JL, et al. American College of Cardiology Foundation; American Heart Association Task Force on Practice Guidelines; American Association for Thoracic Surgery; Society of Cardiovascular Anesthesiologists; Society of Thoracic 
Surgeons. 2011 ACCF/AHA guideline for coronary artery bypass graft surgery: a report of the American College of Cardiology Foundation/American Heart Association Task Force on Practice Guidelines. Developed in collaboration with the American Association for Thoracic Surgery, Society of Cardiovascular Anesthesiologists, and Society of Thoracic Surgeons. Journal of the American College of Cardiology. 2011; 58(24): e123-210. https://doi.org/10.101 $6 / j \cdot j a c c .2011 .08 .009$

[6] Stephens RS, Whitman GJ. Postoperative Critical Care of the Adult Cardiac Surgical Patient: Part II: Procedure-Specific Considerations, Management of Complications, and Quality Improvement. Critical Care of Medicine. 2015; 43: 1995-2014. PMid: 26136101. https://doi.org/10.1097/CCM.0000000000001171

[7] Mazza BF, Gatti MFZ, Macedo RCR, et al. Does a specialized neurological ICU have better performance when compared with a general ICU? Critical Care. 2015; 19(Suppl 2): 48.

[8] Lott JP, Iwashyna TJ, Christie JD, et al. Critical Illness Outcomes in Specialty versus General Intensive Care Units. American Journal of Respiratory and Critical Care Medicine. 2009; 179: 676-683. PMid: 19201923. https://doi .org/10.1164/rccm. 200808-12810C

[9] Santos CA, Oliveira MAB, Brandi AC, et al. Risk factors for mortality of patients undergoing coronary artery bypass graft surgery. Revista Brasileira de Cirurgia Cardiovascular. 2014; 29(4): 513-20. https://doi.org/10.5935/1678-9741.20140073

[10] Hausenloy DJ, Candilio L, Evans R, et al. Remote ischemic preconditioning and outcomes of cardiac surgery. The New England Journal of Medicine. 2015; 373: 1408-17. PMid: 26436207. https : //doi.org/10.1056/NEJMoa1413534

[11] Oliveira EK, Turquetto AL, Tauil PL, et al. Risk factors for prolonged hospital stay after isolated coronary artery bypass grafting. Revista Brasileira de Cirurgia Cardiovascular. 2013; 28: 353-63. https://doi.org/10.5935/1678-9741.20130055

[12] Fang J, Alderman MH. Dissociation of hospitalization and mortality trends for myocardial infarction in the United States from 1988 to 1997. American Journal of Medicine. 2002; 13(3): 208-14. https://doi.org/10.1016/S0002-9343(02)01172-5

[13] De Velasco JA, Llargues E, Fito R, et al. Risk factor prevalence and drug treatment in coronary patients at hospital discharge. Results of a national multicenter registry (3C Program). Revista Espa-ola de
Cardiologia. 2001; 54(2): 159-68. https://doi.org/10.1016/ S0300-8932 (01) 76286-0

[14] Wilson PW, D'Agostino RB, Levy D, et al. Prediction of coronary heart disease using risk factor categories. Circulation. 2008; 9(18): 1837-47. https://doi.org/10.1161/01.CIR.97.18.1837

[15] Eckel RH, Krauss RM. American Heart Association call to action: obesity as a major risk factor for coronary heart disease. AHA Nutrition Committee. Circulation. 1998; 97(21): 2099-100. PMid: 9626167. https://doi.org/10.1161/01.CIR.97.21.2099

[16] Mehran R, Dangas GD, Kobayashi Y, et al. Short- and long-term results after multi vessel stenting in diabetic patients. Journal of the American College of Cardiology. 2004; 43(8): 1348-54. PMid: 15093865. https://doi.org/10.1016/j.jacc.2003.04.004

[17] Natali A, Vichi S, Landi P, et al. Coronary atherosclerosis in Type II diabetes: angiographic findings and clinical outcome. Diabetologia. 2000; 43(5): 632-41. PMid: 10855538. https://doi.org/10.1 $007 / \mathrm{s} 001250051352$

[18] Meadows K, Gibbens R, Gerrard C, et al. Prediction of patient length of stay on the intensive care unit following cardiac surgery: a logistic regression analysis based on the cardiac operative mortality risk calculator, EuroSCORE. Journal of Cardiothoracic and Vascular Anesthesia. 2018. PMid: 29678435. https://doi.org/10.1053/ j.jvca.2018.03.007

[19] Vital JACP, Cruz ICF. Evidence-based practice guidelines for the nursing intervention care of the surgical incision site in the postoperative period of cardiac surgery in the ICU - Systematic Literature Review. Journal of Specialized Nursing Care. 2018; 10(1).

[20] Nguyen YL, Milbrandt EB. Are specialized ICUs so especial? Critical Care Medicine. 2009; 13: 314.

[21] Macias CA, Rosengart MR, Puyana JC, et al. The effects of trauma center care, admission volume, and surgical volume on paralysis after traumatic spinal cord injury. Annals Surgery. 2009; 249: 1017. PMid: 19106669. https://doi.org/10.1097/SLA.0b013e 31818a1505

[22] Diringer MN, Edwards DF. Admission to a neurologic/neurosurgical intensive care unit is associated with reduced mortality rate after intracerebral hemorrhage. Critical Care Medicine. 2001; 29: 635-640. https://doi.org/10.1097/00003246-200103000-00031 